\title{
Multidimensional Scaling as an Aid for the Analytic Network and Analytic Hierarchy Processes
}

\author{
M. A. A. Cox \\ University of Newcastle
}

\begin{abstract}
Graphs are a great aid in interpreting multidimensional data. Two examples are employed to illustrate this point. In the first the many dissimilarities generated in the Analytic Network Process (ANP) are analysed using Individual Differences Scaling (INDSCAL). This is the first time such a procedure has been used in this context. In the second the single set of dissimilarities that arise from the Analytic Hierarchy Process (AHP) are analysed using Multidimensional Scaling (MDS). The novel approach adopted here replaces a complex iterative procedure with a systematic approach that may be readily automated.
\end{abstract}

Key words: Analytic hierarchy process, analytic network process, decision support, individual differences scaling, multidimensional scaling.

\section{Introduction}

It is often said that a picture is worth a thousand words. It is always useful to have diagrams to enhance the interpretation of analytic techniques. In this instance graphical techniques employed by statisticians will be applied to techniques more commonly encountered in management science or operations research.

In recent years many papers have been written examining the Analytic Hierarchy Process (AHP, Saaty, 2001, for example) as an aid to decision making. This has been extended to the Analytic Network Process (ANP, Saaty, 1996). Similarly Multidimensional Scaling (MDS, Cox and Cox, 2001, for example) has been widely employed to assist in data presentation.

Two works have combined these methods. That of Huang et al. (2005) combined the ANP with MDS while Ernstberger (1995) combined the AHP with MDS; these furnish the two examples considered here. The first example benefits from the application of the INDSCAL procedure, while for the second a direct procedure is developed which avoids the iterative scheme previously utilised. 
For those not familiar with the numerical procedures employed here: Analytic Hierarchy Process, Analytic Network Process, Individual Differences Scaling, Non-metric Multidimensional Scaling and Procrustes Analysis. These are now briefly reviewed; the topics are presented in alphabetic order.

\section{Overview Of The Numerical Techniques Employed}

\subsection{Analytic hierarchy process}

The AHP is a method for formalizing decision making where there are a limited number of choices but each has a number of attributes and it my be difficult to formalize some of those attributes. It is a structured technique for dealing with complex decisions. The AHP provides a methodology for structuring a problem, for representing and quantifying its elements, for relating those elements to overall goals, and for evaluating alternative solutions.

A complex problem is decomposed into a hierarchy of simpler sub-problems, each of which can be analysed separately. The elements of the hierarchy can relate to any aspect of the decision problem under consideration. Once the hierarchy is built, the various elements may be analysed. Comparisons are made in pairs. In making the comparisons, assessments of the relative importance of the elements are made.

On completion priorities as numerical values are derived for the alternatives. It is a simple matter to select the optimum choice, or to rank them in order of importance.

The AHP has been used in a large number of applications to provide some structure on a decision making process. Originally it was introduced by Saaty (1977) and more fully described in Saaty (1980) with some nice examples in Wind and Saaty (1980). For a more recent review see Zahedi (1986), while Smith and von Winterfeldt (2004) explore some of the current controversies.

In the AHP procedure the decision maker is required to make pair wise comparisons between $\mathrm{n}$ alternatives based on a ratio scale, the choices are made from the integers between 1 and 9 and their reciprocals $(I=\{1 / 9,1 / 8,1 / 7,1 / 5,1 / 4,1 / 3$, $1 / 2,1,2,3,4,5,6,7,8,9\})$. The resulting decisions are summarised in a square matrix $\left(A: A_{i j}, i=1, \ldots, n ; j=1, \ldots, n\right)$ of dimension $n$. It is reciprocal symmetric that is $a_{i j}=1 / a_{j i}$. The priority vector of the alternatives is the right eigen vector associated with the dominant eigen value $(A \nu=\lambda \nu)$. An alternate approach is to adopt the row geometric mean $\nu_{i}=N^{-1}\left(\prod_{j=1}^{n} a_{i j}\right)^{1 / n}$ with an appropriate normalisation term $\left(N: \sum_{i=1}^{n} \nu_{i}=1\right)$ (Barzilai, 1997). For a detailed review of various alternate approaches to this numerical problem see Choo and Wedley (2004).

For further details and examples see Saaty (1996 and 2001). 


\subsection{Analytic network process}

A more advanced framework for setting priorities known as the ANP method of decision making was developed from the AHP. The ANP differs from the AHP in that it generalises the pair-wise comparison process so that decision models can be built as complex networks which may include the following interconnecting components: decision objectives, criteria, stakeholders, alternatives, scenarios and other factors.

The key concept of the ANP is that influence does not necessarily have to flow downwards, as is the case with the hierarchy in the AHP. Influence can flow between any factors in the network, causing non-linear networks of priorities and alternative choices. The ANP is extremely useful for predictive modelling.

Popular applications of the ANP are decisions where risks and threats are important factors in the decision making process. When success may depend on an understanding of the entire environment, rather than just that of business goals and objectives.

The ANP was proposed by Saaty (Saaty, 1996; Saaty and Vargas, 1998) to overcome the problem of interdependence and feedback between criteria or alternatives. The ANP is the general form of the AHP.

The first phase of the ANP is to compare the criteria in a whole system to form a super matrix. This is achieved using the usual AHP decision criteria. The system can be represented by a network, which represents the interaction of the elements in a cluster (these are the features in the first example), on elements in the same or another cluster. Typically one considers the elements one at a time in one cluster and pair wise comparisons made of the elements in the other clusters to that element. The general form of super matrix, which is a matrix of matrices, (following Huang et al. 2005) is

$$
W=\left(w_{i j}\right)_{n \times n},
$$

where $w_{i j}$ is a matrix associated with the $i$ th cluster $C_{i}$ on the row, and with the $j$ th cluster $C_{j}$ on the column, $C_{m}=\left(e_{m 1}, e_{m 2}, \ldots, e_{m n}\right)^{\prime}, e_{i j}$ denotes the $i$ th element in the $j$ th cluster, $w_{i j}$ is the principal eigenvector of the influence of the elements compared in the $j$ th cluster to the $i$ th cluster. In addition, if the $j$ th cluster has no influence to the $i$ th cluster, then $w_{i j}=0$. Clearly the super matrix $(W)$ is symmetric.

For further details and examples see Saaty and Vargas (1998) and also Huang et al. (2005), who present an example and a fuller discussion. 


\subsection{Individual differences scaling}

INDSCAL is a form of MDS. Its basic assumption is that each individual respond to all the dimensions of the stimuli but may utilize the dimensions in varying degree. Each subject weights the dimensions separately and, maybe, differently.

Carol and Chang (1970) proposed a metric model comprising two spaces: a group stimulus space and a subjects (or individuals) space, both of chosen dimension p. Points in the group stimulus space represent the objects or stimuli, and form an "underlying" configuration. The individuals are represented as points in the subjects space. The coordinates of each individual are the weights required to give the weighted Euclidean distances between the points in the stimulus space, the values that best represent the corresponding dissimilarities for that individual. Hence the acronym INDSCAL INdividual Differences SCALing.

Let the points in the group stimulus space be given by $x_{r t}(r=1, \ldots, n ; t=$ $1, \ldots, p)$. Let the points in the individuals space have coordinates $w_{i t}(i=$ $1, \ldots, N ; t=1, \ldots, p)$. Then the weighted Euclidean distance between stimuli $r$ and $s$, for the $i$ th individual is

$$
d_{r s, j}=\sqrt{\sum_{i=1}^{p} w_{i t}\left(x_{r t}-x_{s t}\right)^{2}}
$$

The individual weights $\left\{w_{i t}\right\}$ and stimuli coordinates $\left\{x_{r t}\right\}$ are then sought by an iterative scheme that best match $\left\{d_{r s, i}\right\}$ to $\left\{\delta_{r s, i}\right.$. For further details and examples see Cox and Cox (2001).

\subsection{Non-metric multidimensional scaling}

MDS is a data visualisation technique for exploring dissimilarities in data. The basic algorithm starts with a symmetric matrix of dissimilarities between items. It assigns a location to each item in a space of an appropriate dimension. The procedure finds a monotonic relationship between the items in the matrix and the Euclidean distance between them. The relationship is typically found using isotonic regression.

The majority of MDS analyses are carried out with $X$ a subset of $R^{P}$, and with $p=2$ in particular. A configuration of points is sought in $R^{P}$ which represent the original objects, such that the distances between the points $\left\{d_{r s}\right\}$ match order wise, as well as possible, the original dissimilarities $\left\{\delta_{r s}\right\}$.

Let the $r$ th point in $X$ have coordinates $x_{r}=\left(x_{r 1}, \ldots, x_{r p}\right)^{\prime}$. 
Let the distance measure for points $r$ and $s$ in $X$, be Euclidean metric

$$
d_{r s}=\sqrt{\sum_{i=1}^{p}\left(x_{r i}-x_{s i}\right)^{2}} .
$$

Define disparities $\left\{\hat{d}_{r s}\right\}$, viewed as a function of the distances $\left\{d_{r s}\right\}$, by

$$
\hat{d}_{r s}=f\left(d_{r s}\right)
$$

where $f$ is a monotonic function such that

$$
\hat{d}_{r s} \leq \hat{d}_{t u} \text { whenever } \delta_{r s}<\delta_{t u} .
$$

Thus the disparities "preserve" the order of the original dissimilarities but allow possible ties in the disparities.

Let the stress function be $S$, where

$$
S=\sqrt{\frac{\sum_{r<s}\left(d_{r s}-\hat{d}_{r s}\right)^{2}}{\sum_{r<s} d_{r s}^{2}}} .
$$

Note the original dissimilarities $\left\{\delta_{r s}\right\}$ only enter into the stress function by defining an ordering for the disparities $\left\{\hat{d}_{r s}\right\}$. The aim is to find a configuration that attains minimum stress.

The approach adopted follows that of Kruskal (1964a, b) and is a complex iterative technique. In particular the monotone function adopted is monotonic least squares regression.

The first step is to choose an initial configuration. Various strategies may be adopted when making this choice, however these frequently result in only locating a local minima. This will often lead to different authors producing conflicting results. To avoid this a number of different randomly selected starting points are employed. Typically the number chosen is selected bearing in mind restrictions on available computer time. For the small problems considered here 1,000 random restarts were employed.

For further details and examples see Cox and Cox (2001).

\subsection{Procrustes analysis}

Procrustes analysis is essentially a method of shape analysis where items located in two clusters are aligned as closely as possible with operations such as dilation, reflection and rotation.

Configurations may be compared employing Procrustes statistics (Mardia et $a l ., 1979)$. The resulting Procrustes statistic takes a value in the range $[0,1]$; 
the procedure seeks the isotropic dilation, reflection and rotation needed to best match the two configurations. A value of zero indicates a perfect match.

Summarizing the steps in a Procrustes analysis where configuration $Y$ is to be matched to configuration $X$ :

1. Subtract the mean vectors for the configurations from each of the respective points in order to have the centroids at the origin.

2. Find the rotation matrix $A=\left(X^{T} Y Y^{T} X\right)^{1 / 2}\left(Y^{T} X\right)^{-1}$ and rotate the $X$ configuration to $X A$.

3. Scale the $X$ configuration by multiplying each coordinate by $\rho$, where $\rho=$ $\operatorname{tr}\left(X^{T} Y Y^{T} X\right)^{1 / 2} / \operatorname{tr}\left(X^{T} X\right)$.

4. Calculate the minimised and scaled value of

$$
R^{2}=1-\left\{\operatorname{tr}\left(X^{T} Y Y^{T} X\right)^{1 / 2}\right\}^{2} /\left\{\operatorname{tr}\left(X^{T} X\right) \operatorname{tr}\left(Y^{T} Y\right)\right\} .
$$

This is known as the Procrustes statistic.

For further details and examples see Cox and Cox (2001).

\section{Example 1 - The Analytic Network Process}

The example employed is taken from Huang et al. (2005). Here six products $\{A, B, C, D, E, F\}$ are to be considered. The decision makers are interested in five features, the price, the package, the location, the function, and the manufacturer. The proximity matrices which summarise expert's opinions are presented in Table 1. Where a value of 1 indicates equal importance and 9 is the most extreme value, often referred to as absolute importance. Intermediate values are interpreted accordingly. The resulting matrix is reciprocal symmetric, $a_{i j}=1 / a_{j i}$. The super matrix is a matrix of matrices, and was given in the original work, it provides the weights (resulting from the eigen analysis) in Table 1. The weights are the appropriately normalised vector corresponding to the unit eigen value of the supermatrix.

This is purely a numerical example developed by Huang et al. (2005) with the aim of assessing the closeness of the products, which represents important information for the firms. Their analysis identifies four groups $\{A, E\},\{B\},\{C\}$ and $\{D, F\}$. Such an interpretation may be debateable. 
Table 1: Proximity matrices

\begin{tabular}{|c|c|c|c|c|c|c|}
\hline Features & & & & & & Weight \\
\hline Price & Product & $\mathrm{A}$ & & & & 0.424 \\
\hline B & 2 & $\mathrm{~B}$ & & & & \\
\hline $\mathrm{C}$ & 2 & 2 & $\mathrm{C}$ & & & \\
\hline $\mathrm{D}$ & 3 & 6 & 7 & $\mathrm{D}$ & & \\
\hline $\mathrm{E}$ & 7 & 8 & 5 & 8 & $\mathrm{E}$ & \\
\hline $\mathrm{F}$ & 5 & 1 & 3 & 7 & 1 & \\
\hline Package & Product & A & & & & 0 \\
\hline B & 8 & $\mathrm{~B}$ & & & & \\
\hline $\mathrm{C}$ & 3 & 5 & $\mathrm{C}$ & & & \\
\hline $\mathrm{D}$ & 5 & 6 & 3 & $\mathrm{D}$ & & \\
\hline $\mathrm{E}$ & 9 & 1 & 2 & 4 & $\mathrm{E}$ & \\
\hline $\mathrm{F}$ & 7 & 1 & 7 & 9 & 1 & \\
\hline Location & Product & A & & & & 0 \\
\hline B & 8 & $\mathrm{~B}$ & & & & \\
\hline $\mathrm{C}$ & 6 & 2 & $\mathrm{C}$ & & & \\
\hline $\mathrm{D}$ & 4 & 9 & 4 & $\mathrm{D}$ & & \\
\hline $\mathrm{E}$ & 6 & 8 & 4 & 4 & $\mathrm{E}$ & \\
\hline $\mathrm{F}$ & 5 & 3 & 1 & 7 & 5 & \\
\hline Function & Product & $\mathrm{A}$ & & & & 0.127 \\
\hline B & 1 & $\mathrm{~B}$ & & & & \\
\hline $\mathrm{C}$ & 3 & 1 & $\mathrm{C}$ & & & \\
\hline $\mathrm{D}$ & 5 & 3 & 7 & $\mathrm{D}$ & & \\
\hline $\mathrm{E}$ & 2 & 7 & 4 & 4 & $\mathrm{E}$ & \\
\hline $\mathrm{F}$ & 2 & 4 & 8 & 6 & 4 & \\
\hline Manufacturer & Product & A & & & & 0.449 \\
\hline B & 4 & B & & & & \\
\hline $\mathrm{C}$ & 8 & 3 & $\mathrm{C}$ & & & \\
\hline $\mathrm{D}$ & 3 & 5 & 3 & $\mathrm{D}$ & & \\
\hline $\mathrm{E}$ & 5 & 2 & 4 & 4 & $\mathrm{E}$ & \\
\hline $\mathrm{F}$ & 9 & 7 & 1 & 6 & 4 & \\
\hline
\end{tabular}

The analysis aims to combine the properties of the five features. As a precursor to this step the similarity between the features is examined. Clearly similar features could be merged.

\section{How Different Are the Features}

Non-metric multidimensional scaling (Kruskal, 1964a and 1964b) is employed on the five proximity matrices from Table 1 (Huang et al., 2005). To ensure a global rather than a local solution was obtained 1,000 random starts were employed. Having obtained the two-dimensional configurations these were compared employing Procrustes statistics (Mardia et al., 1979), these values are summarised in Table 2. 
M. A. A. Cox

Table 2: Procrustes comparison of the vectors for the features

\begin{tabular}{lccccc}
\hline & Price & Package & Location & Function & Manufacturer \\
\hline Price & 0 & 0.589 & 0.952 & 0.765 & 0.803 \\
Package & 0.589 & 0 & 0.503 & 0.712 & 0.696 \\
Location & 0.952 & 0.503 & 0 & 0.669 & 0.701 \\
Function & 0.765 & 0.712 & 0.669 & 0 & 0.984 \\
Manufacturer & 0.803 & 0.696 & 0.701 & 0.984 & 0 \\
\hline
\end{tabular}

A value of zero (as seen on the diagonal of Table 2) indicates a perfect match. Since the smallest off diagonal value exceeds 0.5 it may safely be assumed that the features are viewed as being distinct and all features should be retained.

The previous authors (Huang et al., 2005) took the average of the five proximity matrices, which they then analysed using MDS. This analysis was repeated resulting in the plot in Figure 1; again 1,000 random restarts of the procedure were employed.

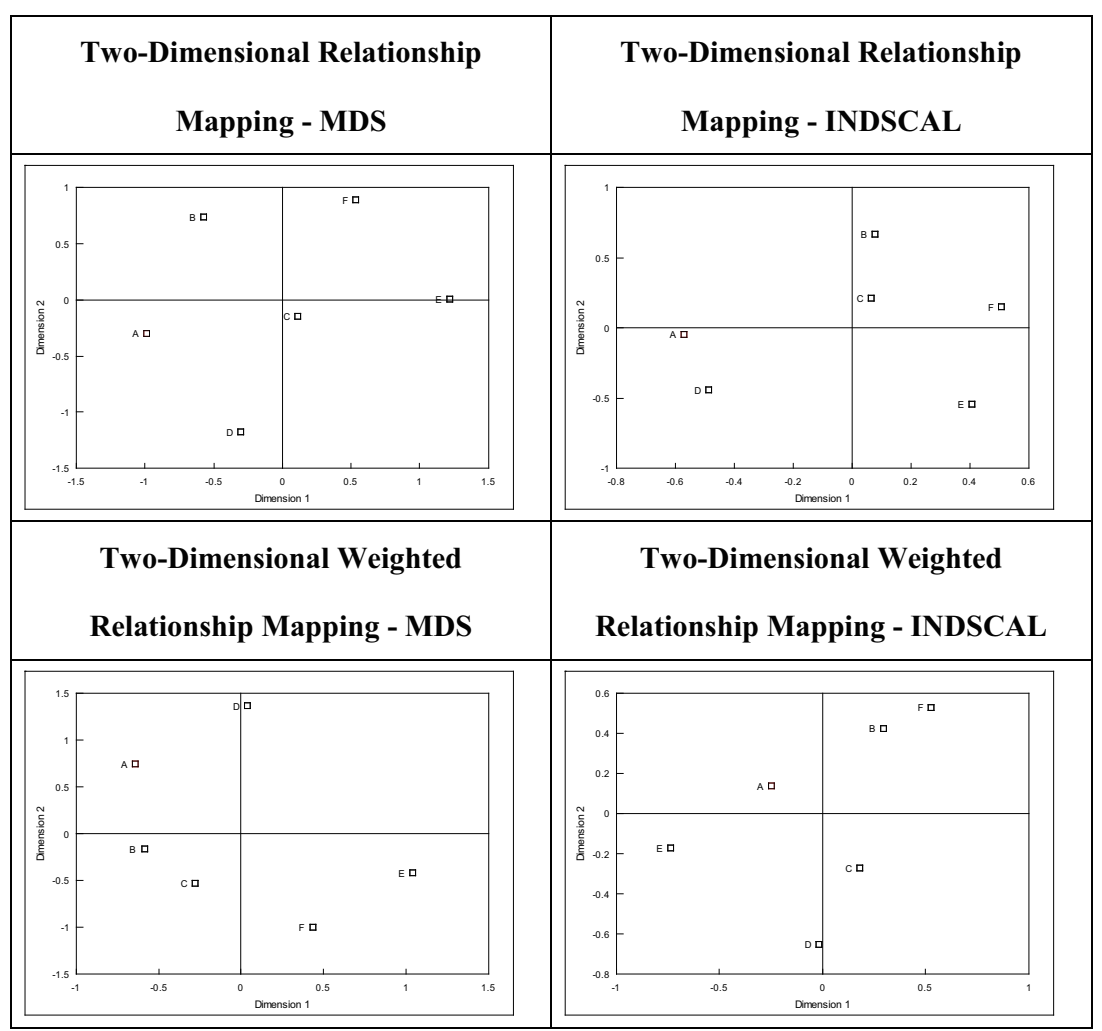

Figure 1: Comparative plots 
For consistency with previous workers the axis have been labelled with numerical scales. This is misleading since a non-metric procedure is used which employs monotone regression. Thus the fit is made to the ordering of the dissimilarities not their magnitude. As pointed out in the Procrustes analysis the result is only unique up to factors of dilation, reflection and rotation.

Unfortunately "averaging" destroys the many differences indicated between the factors by the relatively high Procrustes statistics (Table 2). To preserve and analyse the full set of dissimilarities Individual Differences Scaling (referred to as INDSCAL) as proposed by Carroll and Chang (1970) is employed. This metric model is composed of two spaces, a group stimulus space and a subject's space. Individual subject weights and stimuli coordinates are sought which best match the total set of dissimilarities. The coordinates of the products are displayed in Figure 1.

The Procrustes statistic relating the coordinates in Figure 1 are presented later.

The original authors argued that weights taken from the ANP should have been used when obtaining the average dissimilarities. Repeating the MDS analysis with this constraint produced the coordinates plotted in Figure 1.

Again INDSCAL can be used to preserve the raw data, with each set of dissimilarities being scaled by the weight from the ANP, hence only price, function and manufacturer feature in the analysis, package and location having zero weight. The coordinates are plotted in Figure 1.

As before Procrustes statistics are employed on the results in Figure 1 to assess if the coordinates are truly different, these statistics are summarised in Table 3.

Table 3: Procrustes comparison of the weighted dissimilarities for the products

\begin{tabular}{lcccc}
\hline & MDS & INDSCAL & Weighted MDS & Weighted INDSCAL \\
\hline MDS & 0 & 0.118 & 0.141 & 0.618 \\
INDSCAL & 0.118 & 0 & 0.126 & 0.580 \\
Weighted MDS & 0.141 & 0.126 & 0 & 0.729 \\
Weighted INDSCAL & 0.618 & 0.580 & 0.729 & 0 \\
\hline
\end{tabular}

While they differ the solutions for MDS, Weighted MDS and INDSCAL have some slight similarity, all giving statistics below 0.15 . However it is clear that the weighted INDSCAL solution is very different, since it preserves the most information from the raw data, it is this approach that should be adopted. Thus any conclusions should be drawn from the weighted INDSCAL analysis summarised in Figure 1. The products appear to exhibit three groups, $\{A, E\},\{B, F\}$ and $\{C, D\}$, these pairings would not have been recognised from the other plots (Figure 1$)$. 
M. A. A. Cox

Table 4: AHP matrix and priority vector

\begin{tabular}{lccc}
\hline & Size & Tuition & Student/Faculty \\
\hline Size & 1 & $1 / 4$ & $1 / 3$ \\
Tuition & 4 & 1 & 3 \\
Student/Faculty & 3 & $1 / 3$ & 1 \\
Priority Vector & 0.1199 & 0.6079 & 0.2721 \\
\hline
\end{tabular}

Table 5: Values for each alternative/attribute plus bounds

\begin{tabular}{|c|c|c|c|}
\hline Institution & Size & Tuition & Student/Faculty \\
\hline 1 Anderson University & 667 & 5610 & 6 \\
\hline 2 Ball State University & 14369 & 1767 & 16 \\
\hline 3 Bethel College & 276 & 5452 & 7 \\
\hline 4 Butler University & 2086 & 7418 & 13 \\
\hline 5 Calumet College of St. Jos. & 386 & 2306 & 11 \\
\hline 6 DePauw University & 2320 & 8850 & 15 \\
\hline 7 Earlham College & 1074 & 9696 & 11 \\
\hline 8 Franklin College of Indiana & 711 & 5720 & 14 \\
\hline 9 Goshen College & 963 & 5720 & 14 \\
\hline 10 Grace College & 659 & 4874 & 12 \\
\hline 11 Hanover College & 1046 & 4980 & 13 \\
\hline 12 Huntington College & 461 & 5600 & 17 \\
\hline 13 University of Indianapolis & 1254 & 6440 & 12 \\
\hline 14 Indiana Institute of Tech. & 406 & 5450 & 20 \\
\hline 15 Indiana State University & 9559 & 1760 & 16 \\
\hline 16 Indiana University & 25219 & 1780 & 18 \\
\hline 17 Indiana University S. Bend & 1600 & 1544 & 11 \\
\hline 18 Indiana University S.E. & 2094 & 1246 & 20 \\
\hline 19 IUPUI Fort Wayne & 3758 & 1555 & 12 \\
\hline 20 IUPUI 21 Manchester & 6861 & 1840 & 6 \\
\hline 21 Manchester College & 944 & 6330 & 12 \\
\hline 22 Martin Center College & 115 & 3610 & 8 \\
\hline 23 Purdue University Calumet & 3250 & 1562 & 14 \\
\hline 24 Purdue University & 26185 & 1816 & 12 \\
\hline 25 Rose Hulman Institute & 1300 & 7980 & 14 \\
\hline 26 St. Joseph's College & 798 & 6640 & 15 \\
\hline 27 St. Mary of the Woods & 351 & 6330 & 7 \\
\hline 28 St. Mary's College & 1792 & 7863 & 15 \\
\hline 29 Taylor University & 1478 & 708716 & \\
\hline 30 Tri-State University & 997 & 5958 & 14 \\
\hline 31 University of Evansville & 2227 & 6994 & 14 \\
\hline 32 University of Notre Dame & 7572 & 9650 & 9 \\
\hline 33 University of Southern IN & 2698 & 1440 & 24 \\
\hline 34 Valparaiso University & 2971 & 7092 & 11 \\
\hline 35 Wabash College & 889 & 7545 & 13 \\
\hline Lower bound & 3000 & 0 & 0 \\
\hline Upper bound & 15000 & 2500 & 16 \\
\hline
\end{tabular}

The second example is taken from Ernstberger (1995). 


\section{Example 2 - The Analytic Hierarchy Process}

The example involves the process of choosing a college to attend. Suppose that the choice is restricted to Indiana and it has been determined that the fundamental attributes contributing to this decision include undergraduate enrolment, in-state tuition cost, and the student/faculty ratio. It is also known that this is a simple two level hierarchy with the attributes on the lower level and satisfaction on the upper level. The user is asked to make a pair wise comparison of the relative importance of the attributes on a 9 point scale where 1 means they are equally important and 9 means that choice A (row) is absolutely more important than choice B (column). The matrix of comparisons is assumed to be reciprocal symmetric (see Table 4). The relevant information for al1 potential college choices is provided in Table 5 .

\section{The Data}

In this case there are 3 attributes $(\mathrm{M}=3)$ and $35(\mathrm{~N}=35)$ alternatives. The selected AHP matrix and resulting priority vector (Ernstberger, 1995) are presented in Table 4.

The values corresponding to each alternative and attribute, plus the selected bounds for each attribute (Ernstberger, 1995) are presented in Table 5.

The original integrated method of Ernstberger (1995) will be summarised.

As proposed the procedure was an iterative scheme. Having selected the attributes upon which the decision will be based their relative weights are selected employing the AHP. The user is then asked to impose numerical constraints on the attributes. An integer programming (IP) approach is employed to identify the most relevant choice set. At this stage the user is offered the alternative of employing the AHP or MDS to refine the alternatives. The procedure may now be recommenced.

This procedure requires some quite complex technology (AHP, MDS, IP) plus a great deal of interaction with the user. Some notation will be needed to present the procedure proposed here. The approach is more direct with all operations being achievable with any spreadsheet.

\section{Notation}

Assume there are $N$ alternatives from which the optimum is to be selected based on $M$ attributes. The user makes a pair-wise comparison of the relative importance of the attributes. The AHP is employed to convert these comparisons into a vector of priorities for the attributes $a_{j}: j=1, \ldots, M$. In addition the user must provide upper $u_{j}: j=1, \ldots, M$ and lower $l_{j}: j=1, \ldots, M$ acceptance 
levels on each attribute. The actual values corresponding to each alternative and each attribute must be available $\nu_{i j}: i=1, \ldots, N, j=1, \ldots, M$. To assist in following the procedure the example from Ernstberger (1995) is presented.

Given the same raw information the approach now deviates from that previously employed. The procedure will now be introduced.

Table 6: Transformed values for each alternative/attribute

\begin{tabular}{|c|c|c|c|c|c|}
\hline Institution & Size & Tuition & Student/Faculty & Priority & Rank \\
\hline 1 Anderson University & 0.6671 & 0.2159 & 0.9644 & 0.4737 & 16 \\
\hline 2 Ball State University & 0.8529 & 0.9788 & 0.5449 & 0.8455 & 7 \\
\hline 3 Bethel College & 0.6390 & 0.2410 & 0.9910 & 0.4928 & 15 \\
\hline 4 Butler University & 0.7628 & 0.0445 & 0.7948 & 0.3348 & 26 \\
\hline 5 Calumet College of St. Jos. & 0.6470 & 0.9144 & 0.9215 & 0.8841 & 5 \\
\hline 6 DePauw University & 0.7775 & 0.0065 & 0.6322 & 0.2692 & 35 \\
\hline 7 Earlham College & 0.6956 & 0.0000 & 0.9215 & 0.3341 & 27 \\
\hline 8 Franklin College of Indiana & 0.6702 & 0.1995 & 0.7165 & 0.3966 & 19 \\
\hline 9 Goshen College & 0.6879 & 0.1995 & 0.7165 & 0.3987 & 18 \\
\hline 10 Grace College & 0.6665 & 0.3475 & 0.8641 & 0.5263 & 13 \\
\hline 11 Hanover College & 0.6937 & 0.3263 & 0.7948 & 0.4978 & 14 \\
\hline 12 Huntington College & 0.6524 & 0.2175 & 0.4577 & 0.3350 & 25 \\
\hline 13 University of Indianapolis & 0.7080 & 0.1130 & 0.8641 & 0.3887 & 21 \\
\hline 14 Indiana Institute of Tech. & 0.6484 & 0.2413 & 0.2189 & 0.2840 & 32 \\
\hline 15 Indiana State University & 1.0000 & 0.9793 & 0.5449 & 0.8635 & 6 \\
\hline 16 Indiana University & 0.0664 & 0.9777 & 0.3730 & 0.7038 & 11 \\
\hline 17 Indiana University S. Bend & 0.7313 & 0.9931 & 0.9215 & 0.9421 & 2 \\
\hline 18 Indiana University S.E. & 0.7633 & 1.0000 & 0.2189 & 0.7590 & 9 \\
\hline 19 IUPUI Fort Wayne & 0.8596 & 0.9926 & 0.8641 & 0.9416 & 3 \\
\hline 20 IUPUI 21 Manchester & 0.9771 & 0.9724 & 0.9644 & 0.9707 & 1 \\
\hline 21 Manchester College & 0.6866 & 0.1240 & 0.8641 & 0.3928 & 20 \\
\hline 22 Martin Center College & 0.6272 & 0.6393 & 1.0000 & 0.7359 & 10 \\
\hline 23 Purdue University Calumet & 0.8323 & 0.9922 & 0.7165 & 0.8979 & 4 \\
\hline 24 Purdue University & 0.0000 & 0.9746 & 0.8641 & 0.8276 & 8 \\
\hline 25 Rose Hulman Institute & 0.7111 & 0.0234 & 0.7165 & 0.2945 & 31 \\
\hline 26 St. Joseph's College & 0.6763 & 0.0949 & 0.6322 & 0.3108 & 30 \\
\hline 27 St. Mary of the Woods & 0.6444 & 0.1240 & 0.9910 & 0.4223 & 17 \\
\hline 28 St. Mary's College & 0.7439 & 0.0270 & 0.6322 & 0.2776 & 33 \\
\hline 29 Taylor University & 0.7231 & 0.0624 & 0.5449 & 0.2729 & 34 \\
\hline 30 Tri-State University & 0.6903 & 0.1670 & 0.7165 & 0.3792 & 24 \\
\hline 31 University of Evansville & 0.7717 & 0.0683 & 0.7165 & 0.3290 & 28 \\
\hline 32 University of Notre Dame & 0.9907 & 0.0002 & 0.9910 & 0.3886 & 22 \\
\hline 33 University of Southern IN & 0.8005 & 0.9971 & 0.0000 & 0.7021 & 12 \\
\hline 34 Valparaiso University & 0.8165 & 0.0621 & 0.9215 & 0.3864 & 23 \\
\hline 35 Wabash College & 0.6827 & 0.0388 & 0.7948 & 0.3217 & 29 \\
\hline
\end{tabular}

\section{Procedure}

The values are transformed to reflect the desirability imposed by the user. To this end a normal transform $(\phi)$ is employed, with mean $\mu_{j}=\left(u_{j}+l_{j}\right) / 2$ and standard deviation $\sigma_{j}=\left(u_{j}-l_{j}\right)$. Various alternatives were examined for the standard deviation, such as $\sigma_{j}=\left(u_{j}-l_{j}\right) / 2$, with no major effect on the 
conclusions reached. The chosen transformation has the property of associating relatively high values with observations within the bounds set by the user. Values outside this interval become less attractive. Then the transformed value is $\nu_{i j}^{\prime}=\phi\left(\nu_{i j}: \mu_{j}, \sigma_{j}^{2}\right)$, to complete the transformation each attribute column is further transformed to the range $[0,1]$. This gives the attributes equal weight, so that the priority vector will be applicable. This is performed by identifying the maximum, $\max _{j}=\operatorname{maximum}\left\{\nu_{i j}^{\prime}: i=1, \ldots, N\right\}$, and the minimum, $\min _{j}=\operatorname{mimimum}\left\{\nu_{i j}^{\prime}: i=1, \ldots, N\right\}$, of the transformed data. The final values, employed for the calculation are

$$
\nu_{i j}^{\prime \prime}=\frac{\nu_{i j}^{\prime}-\min _{j}}{\max _{j}-\min _{j}} .
$$

For the example employed here the transformed values are presented in Table 6.

These values may be combined with the priority vector to obtain a priority value, $p_{i}: i=1, \ldots, N$, for each alternative. These values are summarised in Table 6.

The institutions with the highest priority, as presented in Table 6, match those previously identified by Ernstberger (1995).

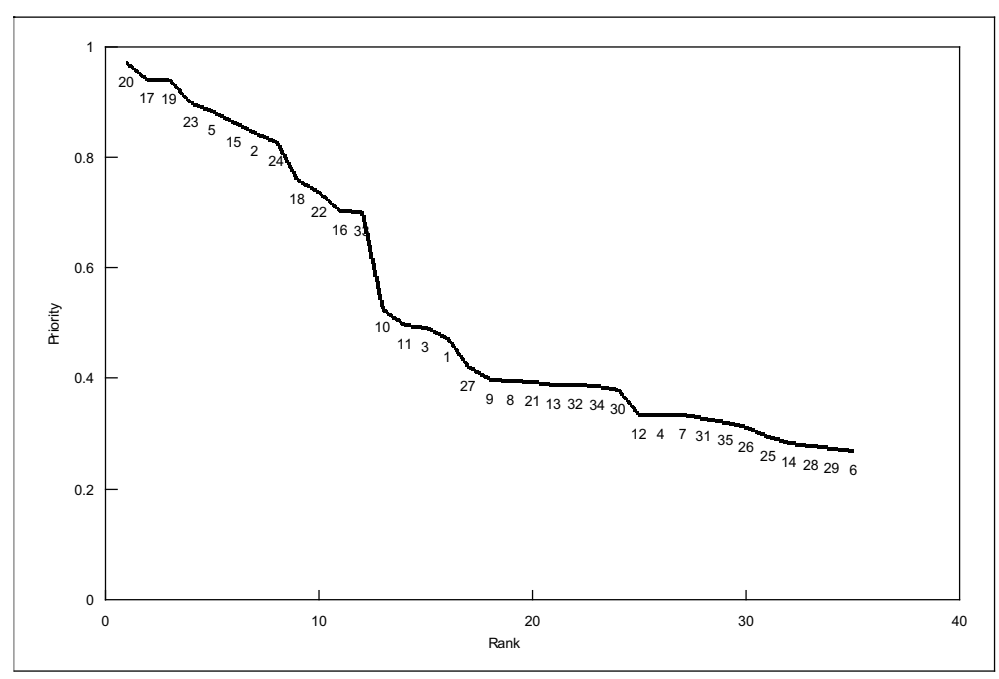

Figure 2: Ranked priority values

A graph of these priority values, Figure 2, makes clear the change in level reflected by the various options. The labels on the figure correspond to the numbers assigned to the institutions. 
The precise shape of this curve, but not apparently the order of the values, is affected by the choice of standard deviation. Similarly the choice of desirability function has little effect.

Obviously this only effectively gives a one-dimensional view of the data. In a similar vein MDS still has a role to play, presenting a more detailed view than that of Figure 2.

\section{Applying Multidimensional Scaling}

To introduce the attribute priorities a weighted Cartesian dissimilarity (Cox and Cox, 2001) is employed. In this case the dissimilarity is $d_{i k}=\sum_{j=1}^{M} a_{j}\left(\nu_{i j}^{\prime \prime}-\right.$ $\left.\nu_{k j}^{\prime \prime}\right)^{2}, i=1, \ldots, N, k=1, \ldots, N$. The resulting plot, Figure 3 , produces a clearer relationship between the various options.

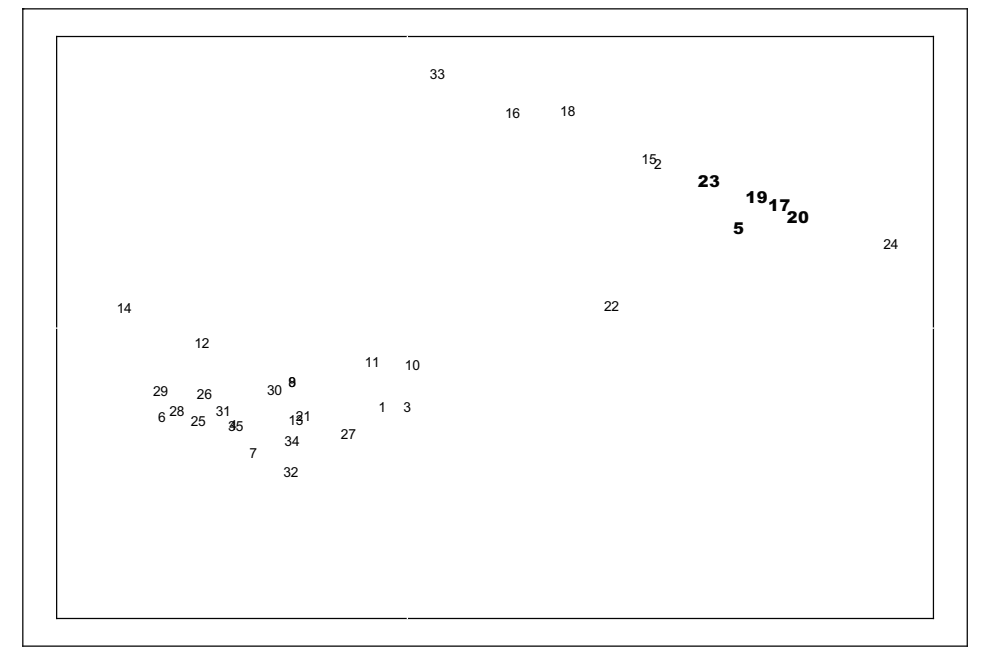

Figure 3: MDS plot of the institutions

Since the procedure only depends on the order of the dissimilarities it has little dependence on the choice of variance and transformation function. It is necessary to refer back to Table 6 to identify the cluster of likely candidates, which have been highlighted in the figure. Thus there are some institutions $\{2,15,22,24\}$ that may be worth a second look, an interesting cluster $\{16,18,33\}$ may also be worth considering, while the remainder may be safely neglected. This degree of interpretation is not possible from Figure 2.

\section{Conclusion}

For the first example considered the authors averaged (or weighted averaged) the dissimilarities and then employed MDS to represent the products. A nat- 
ural consequence of averaging is to blur the difference between the factors. To preserve this difference the INDCSAL procedure is employed. Both procedures result in coordinates for the products that have been demonstrated by Procrustes analysis to be vastly different. The weighted INDSCAL analysis retains the most information about the choices made and is the recommended option.

The procedure presented here for the second example is the conventional AHP with the addition of user-supplied constraints applied to the attribute variables. The AHP priority vector is employed to both generate a priority for each alternative and also dissimilarities between alternatives. The dissimilarities, via MDS, provide a graphical representation of the AHP. The example presented is relatively straightforward, employing only three attributes. The extension of the procedure to problems in much higher dimensions is straightforward and is the strength of the approach used here.

In each case the final decision aid is a simple plot. It is hoped that the ease of interpreting these figures will encourage their use in conjunction with the ANP and AHP.

\section{References}

Barzilai, J. (1997). Deriving weights from pairwise comparison matrices. Journal of the Operational Research Society 48, 1226-1232.

Carroll, J. D. and Chang, J. J. (1970). Analysis of individual differences in multidimensional scaling via an n-way generalization of "Eckart-Young" decomposition. Psychometrika 35, 283-319.

Choo, E. U. and Wedley, W. C. (2004). A common framework for deriving preference values from pairwise comparison matrices. Computers and Operations Research 31, 893-908.

Cox, T. F. and Cox, M. A. A. (2001). Multidimensional Scaling. Chapman and Hall.

Ernstberger, K. W. (1995). A decision support system integrating AHP and MDS to predict choice. Mathematical and Computer Modelling 21, 13-23.

Kruskal, J. B. (1964a). Multidimensional scaling by optimising goodness-of-fit to a nonmetric hypothesis. Psychometrika 29, 1-27.

Kruskal, J. B. (1964b). Nonmetric multidimensional scaling: a numerical method. Psychometrika 29, 115-129.

Huang, J. -J., Tseng, G. -H. and Ong, C. -S. (2005). Multidimensional data in multidimensional scaling using the analytic network process. Pattern Recognition Letters 26, 755-767.

Mardia, K. V., Kent J. T. and Bibby, J. M. (1979). Multivariate Analysis. Academic Press. 
Saaty, T. L. (1977). A scaling method for priorities in hierarchical structures. Journal of Mathematical Psychology 15, 234-281.

Saaty, T. L. (1996). Decision Making with Dependence and Feedback: The Analytic Network Process. RWS publications.

Saaty, T. L. (2001). Decision Making for Leaders. RWS publications.

Saaty, T. L. and Vargas, L. G. (1998). Diagnosis with dependent symptoms: bayes theorem and the analytic hierarchy process. Operations Research 46, 491-502.

Smith, J. E. and von Winterfeldt D. (2004). Decision analysis in management science. Management Science bf 50, 561-574.

Wind Y. and Saaty T. L. (1980). Marketing applications of the analytic hierarchy process. Management Science 26, 641-658.

Zahedi, F. (1986). The analytic hierarchy process - a survey of the method and its applications. Interfaces 16, 96-108.

Received November 28, 2007; accepted December 19, 2007.

M. A. A. Cox

Psychology, Brain and Behaviour

School of Biological Sciences

The Henry Wellcome Building for Neuroecology

University of Newcastle upon Tyne

Framlington Place

Newcastle upon Tyne, NE2 4HH, England

mike.cox@ncl.ac.uk 